\author{
Nr. $174 / 94$ \\ White Noise Approach to Parabolic \\ Stochastic Partial Differential Equations \\ J. Potthoff
}




\title{
WHITE NOISE APPROACH TO PARABOLIC STOCHASTIC PARTIAL DIFFERENTIAL EQUATIONS
}

\author{
J. POTTHOFF \\ Lehrstuhl für Mathematik V \\ Universität Mannheim \\ D-68131 Mannheim \\ Germany
}

ABSTRACT. A certain class of stochastic partial differential equations of parabolic type is studied within white noise analysis.

\section{Introduction}

In [Ch 89], P.L. Chow proposed stochastic partial differential equations of the form

$$
\frac{\partial}{\partial t} u(t, x)-L u(t, x)=\dot{\eta}(t, x) \nabla u(t, x)
$$

as a model for the transport of a substance in a turbulent medium. $L$ is an elliptic second order differential operator acting in the space variable $x \in \mathbb{R}^{d}$. Thus the left hand side of (1.1) describes the diffusion (governed by $L$ ) of the density $u(t, x)$ (at $x$ and at time $t>0)$ of the substance. The right hand side of (1.1) expresses the coupling of the velocity field of the substance to a random field $\dot{\eta}(t, x)$, which models the turbulent velocity field of the medium. This field is the noise of $\eta(t, x)$ which will be assumed to be of the form $\eta(t, x)=\int_{0}^{t} \sigma(s, x) d B(s)$ where $\sigma$ is a deterministic function and $B$ is a $d$-dimensional Brownian motion.

As Chow observed in [Ch 89], generalized random variables arise quite naturally in the discussion of (1.1), and therefore white noise analysis [HK!93] seems to be a natural framework for this type of equation. In fact, as we shall see in Section 4, the coupling of the gradient of $u$ to $\dot{\eta}$ brings a singularity into the corresponding integral equation, which does not allow (at least not in a straightfoward manner) for a formulation in the conventional framework of the Itô calculus. On the other hand, equation (1.1) can indeed be formulated and solved within white noise analysis. In the present paper, explicit solutions for (1.1) will be found and studied for a special choice of $L$ and $\sigma$ (Section 3 ), and existence and uniqueness of weak solutions of (1.1) will be proved in the one-dimensional case (Section 4). The necessary ingredients from white noise analysis will be collected in Section 2. 
I want to remark that a very large amount of work concerning stochastic partial differential equations (SPDE's) within white noise analysis has been done by the group around $\mathrm{B}$. Øksendal in the recent years. The interested reader is referred to $\emptyset \mathrm{ksendal}$ 's contribution in this volume.

In the present article, I did not try to present the most general mathematical results concerning (1.1). Rather, it was my aim to show with a minimal technical effort how classical fixed point theorems combine with some of the tools of white noise analysis $(S-$ transform, characterization theorem, and differential operators), to give existence and uniqueness of solutions. Moreover, I wanted to point out the peculiar behaviour of the solutions of (1.1) with respect to different interpretations ("Itô versus Stratononvich") of the noise term (cf. Section3). A more general theory along the lines of this paper will be published elsewhere [DP 94].

\section{Review of White Noise Analysis}

In this section I give a sketch of white noise analysis which is suited for the applications which are intended in later sections. For a general account on white noise analysis, the interested reader is referred to [HK 93], the references quoted there and to the contribution of L. Streit in this volume.

Consider the white noise probability space $\left(\mathcal{S}^{\prime}(\mathbb{R}), \mathcal{B}, \mu\right)$ where $\mathcal{S}^{\prime}(\mathbb{R})$ is the Schwartz space of tempered distributions, $\mathcal{B}$ is its weak Borel $\sigma$-algebra and $\mu$ is the centered Gaussian measure on $\mathcal{B}$ whose covariance is given by the inner product of $L^{2}(\mathbb{R}, d t$ ) (where $d t$ denotes Lebesgue measure). As is well-known, this probability space is a model for the time derivative of Brownian motion. We denote by $\left(L^{2}\right)$ the $L^{2}$-space over this probability space.

$\left(L^{2}\right)$ contains a dense subspace $(\mathcal{S})$ of smooth random variables which carries a certain metric topology (e.g., [HK 93]). Its dual $(\mathcal{S})^{*}$ is called the space of Hida distributions. $(\mathcal{S})^{*}$, is sufficiently large to contain many generalized random variables which arise in applications. The canonical bilinear pairing between $(\mathcal{S})^{*}$ and $(\mathcal{S})$ will be denoted by $\langle\langle\cdot, \cdot\rangle\rangle$. In order to give elements in $(\mathcal{S})^{*}$ a "visualization", we introduce the $S$-transform: for $\Phi \in(\mathcal{S})^{*}, \xi \in \mathcal{S}(\mathbb{R})$, we set

$$
S \Phi(\xi):=\left\langle\left\langle\Phi,: e^{\langle\cdot, \xi\rangle}:\right\rangle\right\rangle
$$

which makes sense because for $\xi \in \mathcal{S}(\mathbb{R})$, the normalized exponential $\omega \longmapsto: \exp (\langle\omega, \xi\rangle): \equiv$ $\exp \left(\langle\omega, \xi\rangle-\frac{1}{2}|\xi|_{2}^{2}\right), \omega \in \mathcal{S}^{\prime}(\mathbb{R})$, belongs to $(\mathcal{S})$. (We denote by $\langle\cdot, \cdot\rangle$ the dual pairing between $\mathcal{S}^{\prime}(\mathbb{R})$ and $\mathcal{S}(\mathbb{R})$, by $|\cdot|_{2}$ the norm of $L^{2}(\mathbb{R})$.) Indeed, it turns out that the function (2.1) on $\mathcal{S}(\mathbb{R})$ has very nice properties, namely, it is a function of the following type.

Definition 2.1. Consider a mapping $F$ from $\mathcal{S}(\mathbb{R})$ into $\mathbb{C} . F$ is called a $U$-functional if it satisifes the following two properties:

C.1. $F$ is everywhere ray entire on $\mathcal{S}(\mathbb{R})$, i.e., for all $\eta, \xi \in \mathcal{S}(\mathbb{R})$, the mapping $\lambda \longmapsto$ $F(\eta+\lambda \xi), \lambda \in \mathbb{R}$, has an entire extension, 
C.2. there exist constants $K_{1}, K_{2}>0$ and a continuous norm $|\cdot|$ on $\mathcal{S}(\mathbb{R})$ so that for all $\xi \in \mathcal{S}(\mathbb{R}), z \in \mathbb{C}$,

$$
|F(z \xi)| \leq K_{1} \exp \left(K_{2}|z|^{2}|\xi|^{2}\right) .
$$

The space of all such mappings is denoted by $\mathcal{U}$.

Remark. It is interesting to note that $F \in \mathcal{U}$ implies that $F$ has an entire extension to the complexified Schwartz space $\mathcal{S}_{\mathbb{C}}(\mathbb{R})$ (i.e., $F$ extends to a function on $\mathcal{S}_{\mathbb{C}}(\mathbb{R})$ which is continuous and such that C.1 holds for complex $\eta, \xi)$. This has been proved in [KL 94].

The following theorem [PS 91] (cf. also [KL 94]) shows that the elements in $(\mathcal{S})^{*}$ are characterized by their $\mathcal{S}$-transform and $U$-functionals.

Theorem 2.1. The $S$-transform is a bijection from $(\mathcal{S})^{*}$ onto $\mathcal{U}$.

There are many variants, generalizations and extensions of this result. The interested reader is referred to [HK 93, KL 94] and the references quoted there. In passing, I want to mention a result in [BT 94] which says that $\mathcal{U}$ can be equipped with a topology (arising naturally from (2.2)) so that the $S$-transform becomes even a homeomorphism.

Next we discuss differential operators acting on functions on the white noise space.

Let $\varphi$ be a complex valued function on $\mathcal{S}^{\prime}(\mathbb{R})$, and $\theta \in \mathcal{S}^{\prime}(\mathbb{R})$. The Gâteaux derivative $D_{\theta} \varphi(\omega)$ of $\varphi$ in direction $\theta$ at $\omega \in \mathcal{S}^{\prime}(\mathbb{R})$ is given by

$$
D_{\theta} \varphi(\omega):=\left.\frac{d}{d \lambda} \varphi(\omega+\lambda \theta)\right|_{\lambda=0}
$$

whenever the derivative exists. It can be shown (e.g., [HK 93, Chap. 5]) that for all $\theta \in$ $\mathcal{S}^{\prime}(\mathbb{R}), D_{\theta}$ extends to a continuous operator on $(\mathcal{S})$. For the special choice $\theta=\delta_{t}, t \in \mathbb{R}$, this operator will be denoted also by $\partial_{t} . \partial_{t}, t \in \mathbb{R}$, can be considered as a gradient on the white noise space.

By $\partial_{t}^{*}, t \in \mathbb{R}$, we shall denote the adjoint of $\partial_{t}$ which acts on $(\mathcal{S})^{*}$. It is remarkable (though easy to prove) that both operators have very simple intertwining relations with the $S$-transform:

$$
\begin{aligned}
S \partial_{t} \varphi(\xi) & =\frac{\delta}{\delta \xi(t)} S \varphi(\xi), \\
S \partial_{t}^{*} \Phi(\xi) & =\xi(t) S \Phi(\xi),
\end{aligned}
$$

where $\varphi \in(\mathcal{S}), \Phi \in(\mathcal{S})^{*}$, and $\frac{\delta}{\delta \xi(t)}$ denotes the Fréchet functional derivative with respect to $\xi$.

It is well-known (e.g., [HK 93, Chap. 8]) that $\partial_{t}^{*}$ implements a stochastic integral, the Hitsuda-Skorokhod integral, with respect to Brownian motion which extends the Itô integral. Heuristically speaking, the reason is that for $\Phi \in(\mathcal{S})^{*}, \partial_{t}^{*} \Phi$ is equal to the Wick product [HK 93] (cf. also the contribution of B. Øksendal to this volume) $\dot{B}(t) \diamond \Phi$ of white 
noise $\dot{B}(t) \in(\mathcal{S})^{*}$ with $\Phi$ (which is again in $(\mathcal{S})^{*}$ ). If $X$ is a mapping from $\mathbb{R}$ into $(\mathcal{S})^{*}$ such that $t \longmapsto \partial_{i}^{*} X(t)$ is weakly integrable (with respect to Lebesgue measure), then we denote

$$
\begin{aligned}
\int_{\mathbb{R}} X(t) d B(t) & :=\int_{\mathbb{R}} \partial_{t}^{*} X(t) d t \\
& =\int_{\mathbb{R}} X(t) \diamond \dot{B}(t) d t
\end{aligned}
$$

where the last two integrals are in the sense of Pettis and belong to $(\mathcal{S})^{*}$ (e.g., [HK 93]). From (2.4) we obtain

$$
S\left(\int_{\mathbb{R}} X(t) d B(t)\right)(\xi)=\int_{\mathbb{R}} S X(t)(\xi) \xi(t) d t, \quad \xi \in \mathcal{S}(\mathbb{R}) .
$$

Thus the $S$-tranform turns the $d B(t)$-integral into one with respect to $\xi(t) d t$. Relation (2.5) and Theorem 2.2 will be at the basis of our strategy to solve equations of the type (1.1).

\section{Explicit Example}

We consider the following spatially homogeneaus case of the turbulent transport SPDE $(1.1)$

$$
\frac{\partial}{\partial t} u(t, x)-\frac{1}{2} \nu(t) \Delta u(t, x)=\dot{\eta}(t) * \nabla u(t, x),
$$

with $(t, x) \in \mathbb{R}_{+} \times \mathbb{R}$ and $\nu \in L_{\text {loc }}^{1}\left(\mathbb{R}_{+}, d t\right), \nu>0, \sigma \in L_{\text {loc }}^{2}\left(\mathbb{R}_{+}, d t\right)$, and the initial value $u(0, \cdot)=\delta_{0}$, i.e., the Dirac distribution at the origin. (Without loss of generality for the following computations, we consider only the case of one space dimensions.) The * indicates that we shall use as well a Stratonovich interpretation ("* = ") as an Itô interpretation ("* $=\diamond ")$ of (3.1). First we consider (3.1) in Stratonovich sense. Then its Itô form is given by

$$
\frac{\partial}{\partial t} u(t, x)-\frac{1}{2}\left(\nu(t)+\sigma(t)^{2}\right) \Delta u(t, x)=\sigma(t) \dot{B}(t) \diamond \nabla u(t, x) .
$$

Let the $S$-transform of $u$ be denoted by $\widehat{u}$, then (3.2) becomes for $\xi \in \mathcal{S}(\mathbb{R})$ (cf. (2.4)),

$$
\frac{\partial}{\partial t} \widehat{u}(t, x)(\xi)-\frac{1}{2}\left(\nu(t)+\sigma(t)^{2}\right) \Delta \widehat{u}(t, x)(\xi)=\sigma(t) \xi(t) \nabla \widehat{u}(t, x)(\xi) .
$$

Now apply the Fourier transform $\mathcal{F}$ (in the space variable $x$ ), and denote $\mathcal{F} \hat{u}$ by $\tilde{u}$, then we get the following equation

$$
\frac{\partial}{\partial t} \widetilde{u}(t, p)(\xi)+\frac{1}{2}\left(\nu(t)+\sigma(t)^{2}\right) p^{2} \widetilde{u}(t, p)(\xi)=i p \sigma(t) \xi(t) \widetilde{u}(t, p)(\xi),
$$

$(t, p) \in \mathbb{R}_{+} \times \mathbb{R}$. We have $\tilde{u}(0)=(2 \pi)^{-\frac{1}{2}}$, and the solution of (3.4) is readily computed. If we let $\tau(t):=\int_{0}^{t}\left(\nu(s)+\sigma(s)^{2}\right) d s, t \in \mathbb{R}_{+}$, then we obtain

$$
\tilde{u}(t, p)(\xi)=(2 \pi)^{-\frac{1}{2}} \exp \left(-\frac{1}{2} p^{2} \tau(t)+i p \int_{0}^{t} \sigma(s) \xi(s) d s\right)
$$


as the solution of the initial value problem for $\tilde{u}$. It is easy to invert the Fourier transform in (3.5) with the result

$$
\begin{aligned}
S u(t, x)(\xi) & =p_{\tau(t)}\left(x,\left(\sigma_{t}, \xi\right)_{L^{2}(\mathbb{R})}\right) \\
& =(2 \pi \tau(t))^{-\frac{1}{2}} \exp \left(-(2 \tau(t))^{-1}\left(x-\int_{0}^{1} \sigma(s) \xi(s) d s\right)^{2}\right)
\end{aligned}
$$

where $p_{t}(x, y)$ is the usual heat kernel of the Laplacian and $\sigma_{t}=\sigma \cdot 1_{(0, t)}$. In the next step we would like to invert the $S$-transform in (3.6). To this end we use the following more general result. We let $X$. denote the canonical coordinate process on $L^{2}(\mathbb{R})$, i.e., the continuous extension of $X .: \mathcal{S}(\mathbb{R}) \longrightarrow\left(L^{2}\right), X_{\xi}(\omega)=\langle\omega, \xi\rangle, \omega \in \mathcal{S}^{\prime}(\mathbb{R})$, to $L^{2}(\mathbb{R})$.

Lemma 3.1. Let $\lambda_{1}, \lambda_{2} \in \mathbb{C}, f, g \in L^{2}(\mathbb{R})$. Assume that $\left|\lambda_{1}\right||f|_{2}^{-2}<1$. Then for $\xi \in$ $\mathcal{S}(\mathbb{R})$,

$$
S\left(\exp \left(\frac{1}{2} \lambda_{1} X_{f}^{2}+\lambda_{2} X_{g}\right)\right)(\xi)=C\left(\lambda_{1}, \lambda_{2}, f, g\right) \exp \left(\frac{1}{2} a(f, \xi)^{2}+(G, \xi)\right),
$$

where

$$
\begin{aligned}
C\left(\lambda_{1}, \lambda_{2}, f, g\right) & =\left(1-\lambda_{1}|f|_{2}^{2}\right)^{-\frac{1}{2}} \exp \left(\frac{1}{2} \frac{\lambda_{1} \lambda_{2}^{2}}{1-\lambda_{1}|f|_{2}^{2}}(f, g)^{2}+\frac{1}{2} \lambda_{2}^{2}|g|_{2}^{2}\right), \\
a & =\frac{\lambda_{1}}{1-\lambda_{1}|f|_{2}^{2}} \\
G & =\frac{\lambda_{1} \lambda_{2}}{1-\lambda_{1}|f|_{2}^{2}}(f, g) f+\lambda_{2} g .
\end{aligned}
$$

The relations (3.7) help to find the inverse $S$-transforms of exponentials like on the right hand side of (3.7a) via the following. For given $a, f$ and $G$, one finds

$$
\begin{aligned}
\lambda_{1} & =\frac{a}{1+a|f|_{2}^{2}}, \quad \lambda_{2}=1, \\
g & =G-\frac{a(G, f)}{1+a|f|_{2}^{2}} f .
\end{aligned}
$$

The proof of Lemma 3.1 is a straightforward calculation (it seems to be of advantage to decompose $g$ orthogonally in $L^{2}(\mathbb{R})$ along $f$ ). Relations (3.8) follow from trivial algebra.

Corollary 3.2. Let $\tau>0, x \in \mathbb{R}, f \in L^{2}(\mathbb{R})$. Then for $\xi \in \mathcal{S}(\mathbb{R})$,

$$
S p_{\tau}\left(x, X_{f}\right)(\xi)=p_{\tau+|f|_{2}^{2}}\left(x,(f, \xi)_{L^{2}(\mathbb{R})}\right)
$$

(3.9) follows from (3.7) by a simple computation. 
Now we are in position to compute the inverse $S$-transform of (3.6): from (3.9) we can read off that $f=\sigma_{t}$, and with $\gamma(t)=\int_{0}^{t} \nu(s) d s$,

$$
\begin{aligned}
u(t, x) & =p_{\gamma(t)}\left(x, X_{\sigma_{t}}\right) \\
& =(2 \pi \gamma(t))^{-\frac{1}{2}} \exp \left(-(2 \gamma(t))^{-1}\left(x-\int_{0}^{t} \sigma(s) d B(s)\right)^{2}\right) .
\end{aligned}
$$

Of course, it is the same result as in [Ch 89], obtained there in a slightly different way.

Let us return to equation (3.1) and now take $*=\diamond$, i.e., we consider (3.1) in Itô sense. In the preceding calculations this amounts to replacing $\left(\nu(t)+\sigma(t)^{2}\right)$ by $\nu(t)$. Thus, in this case we find for the $S$-transform of the solution instead of (3.6) the formula

$$
v(t, x)(\xi)=p_{\gamma(t)}\left(x,\left(\sigma_{t}, \xi\right)_{L^{2}(\mathbb{R})}\right)
$$

and $v$ solves the equation

$$
\frac{\partial}{\partial t} v(t, x ; \xi)-\frac{1}{2} \nu(t) \Delta v(t, x ; \xi)=\sigma(t) \xi(t) \nabla v(t, x ; \xi)
$$

for every $\xi \in \mathcal{S}(\mathbb{R})$. We can still apply Corollary 3.2 to find the inverse $S$-transform of (3.11), but this time only if $\int_{0}^{t} \nu(s) d s-\int_{0}^{t} \sigma(s)^{2} d s>0$. In that case,

$$
u(t, x)=p_{\kappa(t)}\left(x, \int_{0}^{t} \sigma(s) d B(s)\right)
$$

where $\kappa(t)=\int_{0}^{t} \nu(s) d s-\int_{0}^{t} \sigma(s)^{2} d s$. If $\int_{0}^{t} \nu(s) d s=\int_{0}^{t} \sigma(s)^{2} d s$, we recognize (3.11) as the $S$-transform of Donsker's delta function:

$$
u(t, x)=\delta_{x}\left(\int_{0}^{t} \sigma(s) d B(s)\right) .
$$

In view of the characterization theorem, Theorem 2.2 , we see that also for $\kappa(t)<0,(3.11)$ has an inverse $S$-transform (in $\left.(\mathcal{S})^{*}\right)$. However, it is no longer given by $(3.13)$ : for $\kappa(t)<0$ this expression is no longer real, but since $(3.11)$ is, so must be $u(t, x) \in(\mathcal{S})^{*}$. I have tried a number of times to find an explicit expression for $u(t, x)$ but did not succeed (although it is not hard to write down its chaos expansion).

Note that for $t \in \mathbb{R}_{+}$with $\kappa(t)>0$ we get a very smooth solution: $u(t, x) \in L^{p}(\mu)$ for all $p \geq 1$, which for $\kappa(t) \downarrow 0$ becomes Donsker's delta of $X_{\sigma_{t}}$, and then - as $\kappa(t)$ gets negative - becomes a generalized random variable: one can prove that $u(t, x)$ does not belong to $L^{p}(\mu)$ for any $p \geq 1$. Of course, we can arrange the coefficients $\nu$ and $\sigma$ such that $u(t, x)$ "oscillates" in any given way between being a smooth and a genuinely generalized random field, each time passing through a Donsker delta.

This quite different behaviour of the solution with respect to the interpretation of the multiplication by the noise in (3.1) might be of help concerning questions of modelling 
In the remainder of this section we study in more detail the question, in which sense $u(t, x)=S^{-1} v(t, x)$ is a solution of (3.1) with $*=\diamond$ (which we assume from now on). Obviously we have that for $\zeta \in \mathcal{S}_{\mathbb{C}}(\mathbb{R})$,

$$
v(t, x ; \zeta)=(2 \pi \gamma(t))^{-\frac{1}{2}} \exp \left(-\frac{1}{2 \gamma(t)}\left(x-\int_{0}^{t} \sigma(s) \zeta(s) d s\right)^{2}\right),
$$

is continuously differentiable in $t \in(0,+\infty)$ for all $x \in \mathbb{R}$, and for $t \in(0,+\infty)$ it is $C^{\infty}$ in $x \in \mathbb{R}$, and all derivatives are continuous on $(0,+\infty) \times \mathbb{R}$. It is also obvious that $\frac{\partial}{\partial t} v(t, x ; \cdot), \frac{\partial}{\partial x} v(t, x ; \cdot), \frac{\partial^{2}}{\partial x^{2}} v(t, x ; \cdot)$ belong to $G_{l u}^{2}((0,+\infty) \times \mathbb{R} ; \mathcal{U})$, where this space is defined in Appendix 1. It follows from Lemma A.1.3, that

$$
\begin{aligned}
S^{-1} \frac{\partial}{\partial t} v(t, x) & =\frac{\partial}{\partial t} u(t, x) \\
S^{-1} \frac{\partial}{\partial x} v(t, x) & =\frac{\partial}{\partial x} u(t, x) \\
S^{-1} \frac{\partial^{2}}{\partial x^{2}} v(t, x) & =\frac{\partial^{2}}{\partial x^{2}} u(t, x),
\end{aligned}
$$

where the right hand sides denote weak derivatives in $(\mathcal{S})^{*}$, and they are all weakly continuous functions from $(0,+\infty) \times \mathbb{R}$ into $(\mathcal{S})^{*}$. The fact that $v(t, x ; \zeta)$ solves for every $\xi \in \mathcal{S}(\mathbb{R})$ equation (3.12) means that $u(t, x)$ solves (3.1) weakly on the dense subspace $\mathcal{E}$ which is the (complex) linear span of $\{\exp (<,, \xi>), \xi \in \mathcal{S}(\mathbb{R})\}$. But then $u(t, x)$ solves (3.1) weakly on $(\mathcal{S})$. Now we consider the weak limit of $u(t, x)$ as $t$ falls to zero. Let $f \in \mathcal{S}(\mathbb{R})$ and consider for $\xi \in \mathcal{S}(\mathbb{R})$,

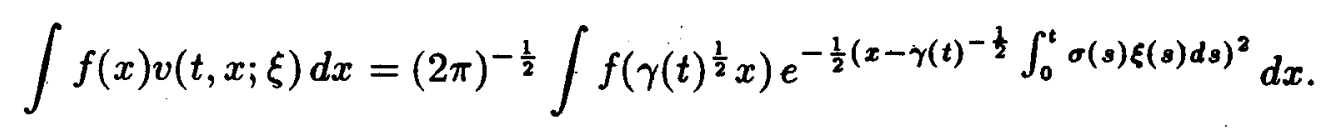

Obviously, the last expression converges to $f(0)$ as $t \downarrow 0$. Moreover, for $t>0, z \in \mathbb{C}, \xi \in$ $\mathcal{S}(\mathbb{R})$, we find

$$
\left|\int f(x) v(t, x ; z \xi) d x\right| \leq\|f\|_{\infty} e^{\frac{1}{2}|z|^{2} \gamma(t)^{-1}\left(\int_{0}^{t} \sigma(u) \xi(u) d u\right)^{2}},
$$

as well as

$$
|v(t, x ; z \xi)| \leq(2 \pi \gamma(t))^{-\frac{1}{2}} e^{\frac{1}{2}|z|^{2} \gamma(t)^{-1}\left(\int_{0}^{t} \sigma(u) \xi(u) d u\right)^{2}} .
$$

In view of Lemma A.1.1, the last estimate shows that for $t>0,\{u(t, x), x \in \mathbb{R}\}$ is bounded in $(\mathcal{S})_{-p}$ for some $p \in \mathbb{N}_{0}$. Moreover, for $t>0, x \longmapsto v(t, x ; \xi)$ is continuous for every $\xi \in \mathcal{S}(\mathbb{R})$. Thus, by Lemma A.1.2, $x \longmapsto u(t, x)$ is weakly continuous, and therefore

$$
\int f(x) u(t, x) d x
$$

exists as a Pettis integral (actually as a Bochner integral), and its $S$-transform is given by

$$
\int f(x) v(t, x ; \xi) d x, \quad \xi \in \mathcal{S}(\mathbb{R})
$$


But this expression converges to $f(0)$ as $t \downarrow 0$ for every $\xi$, which entails that $\int f(x) u(t, x) d x$ converges weakly to $f(0)$ on $\mathcal{E}$. On the other hand the estimate on $\left|\int f(x) v(t, x ; z \xi) d x\right|$ above proves that (cf. Lemma A.1.1) $\int f(x) u(t, x) d x$ is bounded in some $(\mathcal{S})_{-p}, p \in N_{0}$, as $t \downarrow 0$. Hence it converges weakly to $f(0)$. In this sense, $u(t, x)$ admits the initial condition $u(t, \cdot) \rightarrow \delta_{0}$ as $t \downarrow 0$.

In the case of a general equation of type (3.1) our strategy will be to solve an associated integral equation. Let us investigate the concept of a weak solution for the integral equation of $(3.1)$, in order to prepare the next section.

The fundamental solution $q_{t, s}(x, y)$ of $\frac{\partial}{\partial t}-\frac{1}{2} \nu(t) \Delta$ is given by

$$
q_{t, s}(x, y)=p_{\gamma(t)-\gamma(s)}(x, y)
$$

where as before $p_{t}(x, y)$ is the heat kernel. Thus the initial value problem for $(3,1)(*=$ $\diamond)$ with $u(0, \cdot)=u_{0}$ (deterministic again, for simplicity) is reformulated as the integral equation (cf. also [Ch 89])

$$
u(t, x)=\left(P_{\gamma(t)} u_{0}\right)(x)+\int_{0}^{t}\left(P_{\gamma(t)-\gamma(s)} \nabla u(s, \cdot)\right)(x) \sigma(s) \diamond d B(s),
$$

$\left(P_{t} f(x)=\int p_{t}(x, y) f(y) d y\right)$. Taking informally the $S$-transform of (3.15) we arrive at the integral equation

$$
v(t, x ; \xi)=\left(P_{\gamma(t)} u_{0}\right)(x)+\int_{0}^{t}\left(P_{\gamma(t)-\gamma(s)} \nabla v(s, \cdot ; \xi)\right)(x) \sigma(s) \xi(s) d s
$$

Let again $u_{0}=\delta_{0}$, so that the first term on the right hand side of $(3.16)$ becomes $p_{\gamma(t)}(0, x)$. It is not hard (though a bit tedious and certainly no surprise) to verify that $v(t, x ; \xi)$ given in (3.11) solves (3.16). $S^{-1} v(t, x)=u(t, x)$ is the weak solution of (3.15), as we shall argue now. We had already seen in our previous discussion that for $t>0, u(t, x)$ is $C^{1}$ in $t$ and $C^{\infty}$ in $x$, and that $\nabla$ and the $S$-transform commute, i.e. $S^{-1} \nabla v(t, x)=\nabla u(t, x)$. In order to show that the integral $\left(P_{\gamma(t)-\gamma(s)} \nabla u(s, \cdot)\right)(x)$ exists as a Pettis integral in $(\mathcal{S})^{*}$, we estimate $v(s, x ; z \xi)$ for $s>0, x \in \mathbb{R}, z \in \mathbb{C}, \xi \in \mathcal{S}(\mathbb{R})$, as follows:

$$
\begin{aligned}
|\nabla v(s, x ; z \xi)|= & \left|\gamma(s)^{-1}\left(x-z\left(\sigma_{s}, \xi\right)\right)(2 \pi \gamma(s))^{-\frac{1}{2}} \exp \left(-\frac{1}{2} \gamma(s)^{-1}\left(x-z\left(\sigma_{s}, \xi\right)\right)^{2}\right)\right| \\
\leq & \left(2 \pi \gamma(s)^{3}\right)^{-\frac{1}{2}}\left(|x|+|z|\left|\left(\sigma_{s}, \xi\right)\right|\right) \cdot \\
& \quad \exp \left(-\frac{1}{2} \gamma(s)^{-1}\left(x^{2}-2|x||z|\left|\left(\sigma_{s}, \xi\right)\right|-|z|^{2}\left(\sigma_{s}, \xi\right)^{2}\right)\right.
\end{aligned}
$$

where we denoted $\sigma_{s}=\sigma \cdot 1_{[0, s)}$. Now apply the inequality $\pm 2 a b \leq \varepsilon a^{2}+\varepsilon^{-1} b^{2}, \varepsilon>0$, with $\varepsilon=\frac{1}{2}$ :

$$
2|x||z|\left|\left(\sigma_{s}, \xi\right)\right| \leq \frac{1}{2} x^{2}+2|z|^{2}\left(\sigma_{s}, \xi\right)^{2}
$$


so that

$$
\begin{aligned}
|\nabla v(s, x ; z \xi)| & \leq\left(2 \pi \gamma(s)^{3}\right)^{-\frac{1}{2}}\left(|x|+|z|\left|\left(\sigma_{s}, \xi\right)\right|\right) e^{-\frac{1}{4} \gamma(s)^{-1} x^{2}} e^{\frac{3}{2} \gamma(s)^{-1}|z|^{2}\left(\sigma_{o}, \xi\right)^{2}} \\
& \leq(2 \pi)^{-\frac{1}{2}} \gamma(s)^{-1}\left(1+\gamma(s)^{-\frac{1}{2}}|z|\left|\left(\sigma_{s}, \xi\right)\right|\right) e^{\frac{3}{2} \gamma(s)^{-1}|z|^{2}\left(\sigma_{s}, \xi\right)^{2}}
\end{aligned}
$$

The last estimate shows that for $s>0, \nabla v(s, \cdot ;) \in G_{u}^{2}(\mathbb{R} ; \mathcal{U})$ (cf. Appendix 1 ), and so by Lemmas A.1.1, A.1.2, for $s>0, x \longmapsto \nabla u(s, x)$ is weakly continuous and bounded in some $(\mathcal{S})_{-p}, p \in \mathbb{N}_{0}$. Consequently, this mapping is Pettis integrable against a finite measure on the real axis, and hence $\left(P_{\gamma(t)-\gamma(s)} \nabla u(s, \cdot)\right)(x)$ exists as a Hida distribution. Now consider the $d s$-integral in (3.16). For $0 \leq s<t$ we have

$$
\left(P_{\gamma(t)-\gamma(s)} \nabla v(s, \cdot ; z \xi)\right)(x)=-\gamma(t)^{-1}\left(x-z\left(\sigma_{s}, \xi\right)\right) p_{\gamma(t)}\left(x, z\left(\sigma_{s}, \xi\right)\right),
$$

which is obviously in $G_{u}^{2}([0, t] ; \mathcal{U})$ in the $s$-variable for $x \in \mathbb{R}, t>0$. Therefore, by a similar argument as above $(z \sigma(s) \xi(s)$ is trivially taken into account) we have that for $u(t, x)=S^{-1} v(t, x)$ the integral on the right hand side of (3.15) is a well-defined expression in $(\mathcal{S})^{*}$. (3.16) implies that (3.15) holds weakly on a total subspace of $(\mathcal{S})$, and consequently $u(t, x)$ solves $(3.15)$ in weak sense.

\section{The One-Dimensional Case}

Let $\Omega$ denote $\mathbb{R}$ or a domain in $\mathbb{R}$. Consider $D:=[0,1] \times \Omega$ and denote typical elements in $D$ by $(t, x),(s, y)$, etc. where $t, s \in[0,1], x, y \in \Omega$. We are interested in second order differential operators $L$ of the following type in $\Omega$

$$
L f(x)=a(t, x) f^{\prime \prime}(x)+b(t, x) f^{\prime}(x), \quad f \in C^{2}(\Omega),(t, x) \in D .
$$

We assume that $L$ is uniformly elliptic: there exists $\varepsilon>0$ so that for all $(t, x) \in D, a(t, x)>$ $\varepsilon$. We also need a smoothness assumption on the coefficients $a, b$, and for convenience we suppose that they are in $C_{b}^{\infty}(D)$. Under these conditions, the heat equation $\frac{\partial}{\partial t}-L=0$ for $L$ has a fundamental solution $p(t, x ; s, y)$ which admits the following bounds (see, e.g., [Fr 83]): for $t>s \geq 0$,

$$
\begin{aligned}
&|p(t, x ; s, y)| \leq K(t-s)^{-\frac{1}{2}} e^{-\lambda \frac{(x-y)^{2}}{t-d}} \\
&\left|\frac{\partial}{\partial x} p(t, x ; s, y)\right| \leq K(t-s)^{-1} e^{-\lambda \frac{(x-y)^{2}}{t-t}} \\
&\left|\frac{\partial}{\partial y} p(t, x ; s, y)\right| \leq K(t-s)^{-1} e^{-\lambda \frac{(x-y)^{2}}{t-s}}
\end{aligned}
$$

where $K$ and $\lambda$ are appropriate strictly positive constants.

We shall also write for $f \in C_{b}(\Omega)$ and a kernel $q$ on $D \times D$.

$$
\left(Q_{t, s} f\right)(x):=\int_{\Omega} q(t, x ; s, y) f(y) d y,
$$


and for $u \in C_{b}(D)$

$$
\begin{aligned}
(Q u)(t, x) & :=\int_{0}^{t}\left(\int_{\Omega} q(t, x ; s, y) u(s, y) d y\right) d s . \\
& =\int_{0}^{t}\left(Q_{t, s} u(s, \cdot)\right)(x) d s
\end{aligned}
$$

and use similar notations for kernels $p, r$ etc. and their associated integral operators $P_{z, s}, P, R_{t, s}, R$ etc.

Let $\dot{\eta}(t, x)$ denote the (informal) noise of

$$
\eta(t, x):=\int_{0}^{t} \sigma(s, x) d B(s), \quad(t, x) \in D
$$

where $B$ is a Brownian motion, $\sigma \in C_{b}^{1}(D)$.

Our main interest is to study the informal equation

$$
\frac{\partial}{\partial t} u(t, x)-L u(t, x)=\dot{\eta}(t, x) \frac{\partial}{\partial x} u(t, x), \quad(t, x) \in D,
$$

for some random function $u$ on $D$. Obviously, (4.1) needs interpretation, and the examples and computations in Section 3 suggest to allow also for generalized random fields $u(t, x),(t, x) \in D$. The "multiplication" by the noise $\dot{\eta}$ in (4.1) will be interpreted in Hitsuda-Skorokhod sense throughout the present section: $\dot{\eta}(t, x) w \equiv \sigma(t, x) \dot{B}(t) \diamond w$ for any (generalized) random variable $w$. Moreover, I was not able to show that the solution $u$ which will be constructed below is differentiable in $x$. Thus we reformulate this equation in a way that can be given a proper sense. For simplicity, we shall consider here only initial conditions for (4.1) which are given by a deterministic function $u_{0} \in C_{\infty}(\Omega)$. (Here and in the following $C_{\infty}(X), X$ locally compact, is the space of continuous functions (with values in $\mathbb{R}$ or $\mathbb{C}$ ) which vanish outside every compact, i.e., if $f \in C_{\infty}(X)$, then for given $\varepsilon>0$ there exists a compact $C$ in $X$ with $|f(x)|<\varepsilon$ for all $x \in C^{c}$. In particular, every such $f$ is uniformly continuous.) As in [Ch 89], we rewrite (4.1) as an integral equation:

$$
u(t, x)=\int_{\Omega} p(t, x ; 0, y) u_{0}(y) d y+\int_{0}^{t} \int_{\Omega} P(t, x ; s, y) \dot{\eta}(s, y) \frac{\partial}{\partial y} u(s, y) d y d s
$$

or

$$
u(t, x)=\left(P_{t, 0} u_{0}\right)(x)+\int_{0}^{t}\left(P_{t, s} \sigma(s, \cdot) \nabla u(s, \cdot)\right)(x) d B(s) .
$$

Due to the above mentioned difficulties about the differentiability of $u$, we do an informal integration by parts in the convolution with $P$, and write this as

$$
u(t, x)=\left(P_{t, 0} u_{0}\right)(x)+\int_{0}^{t}\left(Q_{t, s}^{\sigma} u(s, \cdot)\right)(x) d B(s),
$$


where $Q_{t, s}^{\sigma}$ has the kernel:

$$
q^{\sigma}(t, x ; s, y)=-\left[p(t, x ; s, y) \frac{\partial}{\partial y} \sigma(s, y)+\left(\frac{\partial}{\partial y} p(t, x ; s, y)\right) \sigma(s, y)\right]
$$

Using the above given estimates on $p(t, x ; s, y)$ and its $y$-derviative, we can bound the kernel by

$$
\left|q^{\sigma}(t, x ; s, y)\right| \leq K\left(|\nabla \sigma|_{\infty}+|\sigma|_{\infty}\right)(t-s)^{-1} e^{-\lambda \frac{(x-y)^{2}}{t-\delta}} .
$$

Here and below, $|\cdot|_{\infty}$ denotes the sup-norm (on $D$ or on $\Omega$, depending on the context). Note that for $s \sim t, q^{\sigma}$ produces a singularity of the type $(t-s)^{-\frac{1}{2}}$, which is not squareintegrable in $s$. (The other factor $(t-s)^{-\frac{1}{2}}$ is for the "normalization" of the Gaussian kernel. Also, the case $L=\Delta$ shows that this singularity does not arise from the lack of a better estimate.) Hence the stochastic integral in (4.3a) cannot be interpreted easily in the usual sense, and we shall interprete in Hitsuda-Skorokhod sense.

We shall say that a mapping $u: D \rightarrow(\mathcal{S})^{*}$ is a weak solution of $(4.1)$ in $C_{\infty}\left(D ;(\mathcal{S})^{*}\right)$, if for every $\varphi \in(\mathcal{S}),\langle u, \varphi\rangle \in C_{\infty}(D)$, and if (4.3a) holds. Here, we define

$$
Q_{t, s}^{\sigma} u(s, \cdot)(x)
$$

as the element in $(\mathcal{S})^{*}$ which for $\varphi \in(\mathcal{S})$ is given by $(t>s)$

$$
\left\langle Q_{t, s}^{\sigma} u(s, \cdot)(x), \varphi\right\rangle=\int_{\Omega} q^{\sigma}(t, x ; s, y)\langle u(s, y), \varphi\rangle d y
$$

i.e., by the integral operator $Q_{t, s}^{\sigma}$ with an integral in the sense of Pettis.

We study (4.3a) by taking its $S$-transform. Let $\xi \in \mathcal{S}(\mathbb{R})$ and $S u(t, x) \equiv \widehat{u}(t, x)$. Then the $S$-transform of (4.3a) at $\xi$ is

$$
\widehat{u}(t, x)(\xi)=\left(P_{t, 0} u_{0}\right)(x)+\int_{0}^{t}\left(Q_{t, s}^{\sigma} \widehat{u}(s, \cdot)(\xi)\right)(x) \xi(s) d s .
$$

It will be useful to consider (4.5) for fixed $\xi$ but to generalize to $\xi \in \mathcal{S}_{\mathbb{C}}(\mathbb{R})$ and a more general "initial" value $v_{0}(t, x)$. I.e., we consider (the $\xi$-dependence of $v$ being suppressed to simplify the notation): $v_{0} \in C_{\infty}(D)$ and the equation

$$
v(t, x)=v_{0}(t, x)+\int_{0}^{t}\left(R_{s, t} v(x, \cdot)\right)(x) \xi(s) d s
$$

for fixed $\xi \in \mathcal{S}_{\mathbb{C}}(\mathbb{R})$ and a kernel $r(t, x ; s, y)$ of $R_{s, t}$ which is continuous on $D \times D \backslash$ \{diagonal $\}$ (the diagonal in $D \times D$ is the subset $\{(t, x ; s, y), t=s, x=y\}$ ), which satisfies

$$
|r(t, x ; s, y)| \leq K_{1}(t-s)^{-1} e^{-\lambda \frac{(x-y)^{2}}{1-\theta}},
$$


where $K_{1}$ is some constant. We are going to solve (4.7) by a fixed point method on the (complex) space $C_{\infty}(D)$. Due to the facts that the kernel $r$ has an integrable singularity, and that it falls off rapidly at infinity, it follows by standard estimates that $R_{s, t}$ maps $C_{\infty}(D)$ into itself. Thus the mapping $T_{\xi}$ defined by

$$
\left(T_{\xi} w\right)(t, x):=v_{o}(t, x)+\int_{0}^{t}\left(R_{s, t} w(s, \cdot)\right)(x) \xi(s) d s
$$

maps $C_{\infty}(D)$ into itself, and the solution of (4.6) is a fixed point of $T_{\xi}$. In order to show that $T_{\xi}$ has a unique fixed point in $C_{\infty}(D)$, we equip $C_{\infty}(D)$ with the following norm

$$
|w|_{\xi, \infty}:=\sup _{(t, x) \in D}|w(t, x)| \psi_{1, \rho(\xi)}^{-1}(t),
$$

where $\psi_{1, \rho(\xi)}>0$ is the unique solution of

$$
\psi_{1, \rho(\xi)}(t)=1+\rho(\xi) \int_{0}^{t}(t-s)^{-\frac{1}{2}} \psi_{1, \rho(\xi)}(s) d s
$$

with $\rho(\xi):=1+K_{2}|\xi|_{\infty}, K_{2}$ some constant which we choose below. In Appendix 2 it is shown that $|\cdot|_{\xi, \infty}$ is equivalent to the usual sup-norm. In particular, $\left(C_{\infty}(D),|\cdot|_{\xi, \infty}\right)$ is a Banach space, and we only need to prove that $T_{\xi}$ is a strict contraction with respect to $|\cdot|_{\xi, \infty}$. But this follows from the following estimation for $v, w \in C_{\infty}(D)$ : let $(t, x) \in D$, then

$$
\begin{aligned}
\mid T_{\xi} v(t, x) & -T_{\xi} w(t, x) \mid= \\
& =\left|\int_{0}^{t} R_{t, s}(v(s, \cdot)-w(s, \cdot))(x) \xi(s) d s\right| \\
& \leq K_{1}|\xi|_{\infty} \int_{0}^{t} \int_{\Omega}(t-s)^{-1} e^{-\lambda \frac{(x-y)^{2}}{t-s}}|v(s, y)-w(s, y)| d y d s \\
& \leq K_{1}|\xi|_{\infty} \int_{0}^{t}(t-s)^{-\frac{1}{2}}|v(s, \cdot)-w(s, \cdot)|_{\infty} d s \int_{\mathbb{R}}(t-s)^{-\frac{1}{2}} e^{-\lambda \frac{(x-y)^{2}}{t-s}} d y \\
& =K_{1} \sqrt{\frac{\pi}{\lambda}}|\xi|_{\infty} \int_{0}^{t}(t-s)^{-\frac{1}{2}}|v(s, \cdot)-w(s, \cdot)|_{\infty} d s \\
& =K_{1} \sqrt{\frac{\pi}{\lambda}}|\xi|_{\infty} \int_{0}^{t}(t-s)^{-\frac{1}{2}} \psi_{1, \rho(\xi)}(s) \psi_{1, \rho(\xi)}^{-1}(s)|v(s, \cdot)-w(s, \cdot)|_{\infty} d s \\
& \leq K_{1} \sqrt{\frac{\pi}{\lambda}}|\xi|_{\infty}|v-w|_{\xi, \infty} \int_{0}^{t}(t-s)^{-\frac{1}{2}} \psi_{1, \rho(\xi)}(s) d s \\
& =K_{1} \sqrt{\frac{\pi}{\lambda}}|\xi|_{\infty}|v-w|_{\xi, \infty} \rho(\xi)^{-1}\left(\psi_{1, \rho(\xi)}(t)-1\right) \\
& \leq K_{1} \sqrt{\frac{\pi}{\lambda}}|\xi|_{\infty} \rho(\xi)^{-1}|v-w|_{\xi, \infty} \psi_{1, \rho(\xi)}(t),
\end{aligned}
$$


where we used $\psi_{1, \rho(\xi)}>0$ (cf. Lemma A.2.2), and equation (4.10) in the last equality. Thus we get

$$
\left|T_{\xi} v-T_{\xi} w\right|_{\xi, \infty} \leq K_{1} \sqrt{\frac{\pi}{\lambda}}|\xi|_{\infty} \rho(\xi)^{-1}|v-w|_{\xi, \infty}
$$

and it suffices to choose $K_{2}=K_{1} \sqrt{\frac{\pi}{\lambda}}$ so that $T_{\xi}$ is a strict contraction. (Recall that $K_{1}=K\left(|\nabla \sigma|_{\infty}+|\sigma|_{\infty}\right)$ in our original problem.) Hence we have proved the following

Lemma 4.1. For every $\xi \in \mathcal{S}_{\mathbb{C}}(\mathbb{R}),(4.6)$ has a unique solution $\widehat{u}(t, x ; \xi)$, which - as a function of $(t, x) \in D$ - belongs to $C_{\infty}(D)$.

Next we study $\widehat{u}$ as a function of $\xi \in \mathcal{S}_{\mathbb{C}}(\mathbb{R})$ in more detail. First we derive an estimate of Gronwall type. Similarly as in the proof that $T_{\xi}$ is a contraction, we can bound $\widehat{u}(t, x)(\xi)$ as follows:

$$
\begin{aligned}
|\widehat{u}(t, x)(\xi)| & \leq\left|v_{0}\right|_{\infty}+K_{1}|\xi|_{\infty} \int_{0}^{t}(t-s)^{-\frac{1}{2}}|\widehat{u}(s, \cdot)(\xi)|_{\infty} d s \int_{\mathbb{R}}(t-s)^{-\frac{1}{2}} e^{-\lambda \frac{(x-y)^{2}}{t-\bullet} d y} \\
& =\left|v_{0}\right|_{\infty}+K_{1} \sqrt{\frac{\pi}{\lambda}}|\xi|_{\infty} \int_{0}^{t}(t-s)^{-\frac{1}{2}}|\widehat{u}(s, \cdot)(\xi)|_{\infty} d s
\end{aligned}
$$

and hence we have

$$
|\widehat{u}(t, \cdot)(\xi)|_{\infty} \leq\left|v_{o}\right|_{\infty}+K_{1} \sqrt{\frac{\pi}{\lambda}}|\xi|_{\infty} \int_{0}^{t}(t-s)^{-\frac{1}{2}}|\widehat{u}(s, \cdot)(\xi)|_{\infty} d s .
$$

Since $(t, x) \longmapsto \widehat{u}(t, x)(\xi)$ is for every $\xi \in \mathcal{S}_{\mathbb{C}}(\mathbb{R})$ in $C_{\infty}(D)$, it follows that $t \longmapsto$ $|\widehat{u}(t, \cdot)(\xi)|_{\infty}$ is continuous on $[0,1]$. Thus we may apply Lemma A.2.4 in Appendix 2 to conclude the following result.

Lemma 4.2. For every $t \in[0,1], \xi \in \mathcal{S}_{\mathbb{C}}(\mathbb{R})$, the unique solution of $(4.6), \widehat{u}(t, x)(\xi)$, admits the following bound

$$
|\widehat{u}(t, \cdot)(\xi)|_{\infty} \leq\left|v_{0}\right|_{\infty}\left(1+K_{1} \pi|\xi|_{\infty} \sqrt{\frac{t}{\lambda}}\right) \exp \left(K_{1}^{2} \pi^{2} \frac{t}{\lambda}|\xi|_{\infty}^{2}\right)
$$

In particular, for some constants $K_{3}, K_{4}>0$, one has

$$
|\widehat{u}(\xi)|_{\infty} \leq K_{3}\left|v_{0}\right|_{\infty} e^{K_{4}|\xi|_{\infty}^{2}}
$$

Now let $\xi$ be of the form $\xi=\eta+z \zeta, \eta, \zeta \in \mathcal{S}(\mathbb{R}), z \in \mathbb{C}$, and write

$$
\widehat{u}(t, x)(\xi) \equiv \widehat{u}(t, x ; z) .
$$

We are interested in the analytic properties of $z \longmapsto \widehat{u}(t, x ; z)$. If $z=z_{1}+i z_{2}, z_{1}, z_{2} \in \mathbb{R}$, it is not hard to use the same methods as before to show that

$$
\frac{\partial}{\partial z_{i}} \widehat{u}(t, x ; z), \quad i=1,2,(t, x) \in D
$$


exist. But then $\frac{\partial}{\partial \bar{z}} \widehat{u}(t, x ; z)$ satisfies

$$
\frac{\partial}{\partial \bar{z}} \widehat{u}(t, x ; z)=\int_{0}^{t}\left[Q_{t, s}^{\sigma}\left(\frac{\partial}{\partial \bar{z}} \widehat{u}(s, \cdot ; z)\right)(\xi)\right](x) \xi(s) d s
$$

and hence Lemma 4.2 implies that $\frac{\partial}{\partial \bar{z}} \widehat{u}(t, x ; z)=0$. In other words:

Lemma 4.3. For all $(t, x) \in D, \eta, \zeta \in \mathcal{S}(\mathbb{R})$, the mapping

$$
z \longmapsto \widehat{u}(t, x)(\eta+z \zeta)
$$

is entire.

By Theorem 2.2, it follows that $\widehat{u}(t, x)$ is the $S$-transform of an element $u(t, x) \in(\mathcal{S})^{*}$. Using the bound (4.12) and the continuity of $(t, x) \mapsto \widehat{u}(t, x ; \xi), \xi \in \mathcal{S}(\mathbb{R})$, we can now apply the same reasoning as at the end of Section 3 to obtain the final result:

Theorem 4.4. For every initial condition $u_{0} \in C_{\infty}(D)$, equation (4.1) has a unique weak solution in $C_{\infty}\left(D ;(\mathcal{S})^{*}\right)$.

It is clear that Theorem 4.4 can be generalized in many ways. For instance, one can choose more general initial conditions, the conditions on the coefficients $a, b, \sigma$, can be improved, the method of proof generalizes to $d$-dimensional domains and certain nonlinear terms can be added to (4.1). Moreover, the arguments leading to Theorem 4.4 can also be used to discuss other equations, such as anticipating stochastic differential and stochastic Volterra equations (cf. also [CL 93]). All this will be worked out in forthcoming papers [DP 94].

\section{Appendix 1: Weak Differentiability}

In this appendix we establish some results concerning the calculus of Hida distributions which depend on a variable in $\mathbb{R}^{d}$. The notions of continuity and differentiability used here are formulated in the weak topologhy of $(\mathcal{S})^{*}$, although most of what is stated here holds also (with a little more care in the arguments) for the strong topology.

Let $O$ be an open set in $\mathbb{R}^{d}$, and consider a mapping $f$ from $O$ into $(\mathcal{S})^{*}$. Let $x_{0} \in O$ and $k \in\{1, \ldots, d\}$. We shall say that $f$ has a weak partial derivative in direction $k$ at $x_{0}$, if there exists an element in $(\mathcal{S})^{*}$ denoted by $\left(D_{k} f\right)\left(x_{0}\right)$, so that

$$
h^{-1}\left(f\left(x_{0}+h e_{k}\right)-f\left(x_{0}\right)\right)
$$

converges weakly in $(\mathcal{S})^{*}$ to $\left(D_{k} f\right)\left(x_{0}\right)$ as $h$ converges to zero. ( $e_{k}$ is the unit vector in $\mathbb{R}^{d}$. in direction $k$.) If $f$ has at all points $x \in O$ weak partial derivatives in all directions $k=1, \ldots, d$, such that for all $k=1, \ldots, d, x \longmapsto D_{k} f(x)$ is weakly continuous from $O$ into $(\mathcal{S})^{*}$, we say that $f$ is weakly continuously differentiable in $O$. The space of all such mappings will be denoted by $C_{w}^{1}\left(O ;(\mathcal{S})^{*}\right)$. Obviously, if $f \in C_{w}^{1}\left(O ;(\mathcal{S})^{*}\right)$, then for all $\varphi \in(\mathcal{S}), x \longmapsto\langle f(x), \varphi\rangle$ belongs to $C^{1}(O)$. 
Assume that for all $x \in O, k=1, \ldots, d, D_{k} f(x) \in(\mathcal{S})^{*}$ exists. Then we can repeat the preceding considerations: for $n \in \mathbb{N}$ we shall say that $f: O \rightarrow(\mathcal{S})^{*}$ is $n$ times weakly continuously differentiable in $U$, if for every multi-index $\alpha=\left(\alpha_{1}, \ldots, \alpha_{d}\right)$ of length $|\alpha|=\alpha_{1}+\ldots+\alpha_{k} \leq n$, the weak partial derivative $D_{f}^{\alpha}(x)=\left(D_{1}^{a_{1}} \cdots D_{d}^{\alpha_{d}} f\right)(x)$ exists for all $x \in O$, and is a weakly continuous function in $O$ with values in $(\mathcal{S})^{*}$. The class of all such mappings is denoted by $C_{w}^{n}\left(O ;(\mathcal{S})^{*}\right) . C_{w}^{0}\left(O ;(\mathcal{S})^{*}\right) \equiv C_{w}\left(O ;(\mathcal{S})^{*}\right)$ is the space of all weakly continuous mappings from $O$ into $(\mathcal{S})^{*}, C_{w}^{\infty}\left(O ;(\mathcal{S})^{*}\right)$ the space of those $f: O \rightarrow(\mathcal{S})^{*}$, such that for every $n \in \mathbb{N}, f \in C_{w}^{n}\left(O ;(\mathcal{S})^{*}\right)$.

In the following we shall give criteria for when a $U$-functional which depends on $x \in O$ has an inverse $S$-transform in $C_{w}^{n}\left(O ;(\mathcal{S})^{*}\right)$. First we make the following definitions.

Let $v$ be a mapping from $O$ into the space $\mathcal{U}$ of $U$-functionals (cf. Section 2). We say that $v$ is locally of uniform growth less than two, if every $x \in O$ has a neighborhood $O_{x}$ in $O$, such that there exist $K_{1}, K_{2}>0$ and $p \in \mathbb{N}_{0}$, so that for all $x^{\prime} \in O_{x}, \xi \in \mathcal{S}(\mathbb{R}), z \in \mathbb{C}$, we have the estimate

$$
\left|v\left(x^{\prime} ; z \xi\right)\right| \leq K_{1} e^{K_{2}|z|^{2}|\xi|_{2, p}^{2}} .
$$

The space of all mappings from $O$ into $\mathcal{U}$ which are locally of uniform growth less than two will be denoted by $G_{l u}^{2}(O ; \mathcal{U})$. If $O_{x}$ above can be chosen as $O$ for every $x \in O$, then we simply say that $v$ is uniformly of growth less than two, and denote the corresponding space by $G_{u}^{2}(O ; \mathcal{U})$.

The following is a re-statement of a result in [PS 91] (cf. also [KL 94]).

Lemma A.1.1. Assume that $v \in G_{u}^{2}(O ; \mathcal{U})$. Then the family $\left\{f(x)=S^{-1} v(x) ; x \in O\right\}$ of Hida distributions is bounded in $(\mathcal{S})_{-q}$ for some $q \in \mathbb{N}_{0}$.

Next we prove a criterium for weak continuity of a family of Hida distributions. Denote by $C_{l u}(O ; \mathcal{U})$ the subspace of $G_{l u}^{2}(O ; \mathcal{U})$ consisting of those $v$ so that for all $\xi \in \mathcal{S}(\mathbb{R}), x \longmapsto$ $v(x ; \xi)$ is continuous from $O$ into $\mathbb{C}$.

Lemma A.1.2. If $v \in C_{l u}(O ; \mathcal{U})$, then $S^{-1} v \in C_{w}\left(O ;(S)^{*}\right)$.

Proof. Let $x \in O$. Then $v \in G_{u}^{2}\left(O_{x}, \mathcal{U}\right)$ for some neighborhood $O_{x}$ of $x$. By Lemma A.1.1 we know that $\left\{f\left(x^{\prime}\right) ; x^{\prime} \in O_{x}\right\}$ is bounded in some $(\mathcal{S})_{-q}$, say by $M>O$.

Let $\mathcal{E}$ be the (complex) linear span of $\{\exp (\langle\cdot, \xi)) ; \xi \in \mathcal{S}(\mathbb{R})\}$, which is a dense subspace of $(\mathcal{S})$. By hypothesis, for every $\psi \in \mathcal{E}$, the mapping $x \longmapsto\langle f(x), \psi\rangle$ is continuous. Now let $\varepsilon>0$ and $\varphi \in(\mathcal{S})$ be given. Choose $\psi \in \mathcal{E}$ so that $\|\varphi-\psi\|_{2, q}<\frac{\varepsilon}{4 M}$. Choose $\delta>0$ small enough, so that $\left\{x^{\prime} ;\left|x-x^{\prime}\right|<\delta\right\} \subset O_{x}$, and such that $\left|x-x^{\prime}\right|<\delta$ implies

$$
\left|\langle f(x), \psi\rangle-\left\langle f\left(x^{\prime}\right), \psi\right\rangle\right|<\frac{\varepsilon}{2}
$$

Then

$$
\begin{aligned}
\left|\langle f(x), \varphi\rangle-\left\langle f\left(x^{\prime}\right), \varphi\right\rangle\right| & \leq\left|\langle f(x), \psi\rangle-\left\langle f\left(x^{\prime}\right), \psi\right\rangle\right|+2 M\|\varphi-\psi\|_{2, q} \\
& <\varepsilon .
\end{aligned}
$$


Now we turn to weak differentiability. Let $n \in \mathbb{N}$ and consider the subspace $C_{l u}^{n}(O ; \mathcal{U})$ of $G_{l u}^{2}(O ; \mathcal{U})$ which consists of those $v$ such that: (i) for every $\zeta \in \mathcal{S}_{\mathbb{C}}(\mathbb{R}), x \longmapsto v(x ; \zeta)$ belongs to $C^{n}(O)$, and (ii) for every multiindex $\alpha,|\alpha| \leq n, D^{\alpha} v \in G_{l u}^{2}(O ; \mathcal{U})$.

Lemma A.3. Assume that $v \in C_{l u}^{n}(O ; \mathcal{U}), n \in \mathbb{N}$ or $n=+\infty$. Then $S^{-1} v \in C_{w}^{n}\left(O ;(\mathcal{S})^{*}\right)$.

Proof. We only prove the case $n=1$, the general case follows by iteration. Denote $D_{k} f:=S^{-1} D_{k} v, k=1, \ldots, d$. By assumption and Lemma A.1.2 we know that $D_{k} f \in$ $C_{w}\left(O ;(\mathcal{S})^{*}\right)$. It remains to show that for all $x \in O, \varphi_{\in} \in(\mathcal{S}), k=1, \ldots, d$, we have

$$
\lim _{h \rightarrow 0}\left[h^{-1}\left(\left\langle f\left(x+h e_{k}\right), \varphi\right\rangle-\langle f(x, \varphi)\rangle\right)-\left\langle D_{k} f(x), \varphi\right\rangle\right]=0,
$$

and our hypothesis implies that this holds for all $\varphi \in \mathcal{E}$. We can therefore conclude the argument as in the proof of Lemma A.1.2, if we can show that there exists $\delta>0$, so that

$$
\left\{h^{-1}\left(f\left(x+h e_{k}\right)-f(x)\right) ; 0<|h|<\delta\right\}
$$

is bounded in $(\mathcal{S})_{-q}$ for some $q \in \mathbb{N}_{0}$.

Let $x \in O$ and $O_{x, k}$ a neighborhood of $x$ so that $D_{k} v \in G_{u}^{2}\left(O_{x, k} ; \mathcal{U}\right)$. Choose $\delta>0$ small enough such that $0<|h|<\delta$ implies $x+h e_{k} \in O_{x, k}$. Let $z \in \mathbb{C}, \xi \in \mathcal{S}(\mathbb{R})$, then

$$
\left|h^{-1}\left(v\left(x+h e_{k} ; z \xi\right)-v(x ; z \xi)\right)\right|=\left|\left(D_{k} v\right)\left(x^{\prime} ; z \xi\right)\right|
$$

for some $x^{\prime} \in O_{x, k}$ by the mean-value theorem. But the last expression is of uniform growth less than two on $O_{x, k}$. Thus $\left\{h^{-1}\left(f\left(x+h e_{k}\right)-f(x)\right) ; 0<|h|<\delta\right\}$ is bounded in $(\mathcal{S})_{-q}$, for some $q \in \mathbb{N}_{0}$, by Lemma A.1.1.

Remark. The criteria for weak continuity and for weak continuous differentiability are not quite "symmetric" with respect to the role of the Schwartz space variable. Perhaps one can combine the results in [KL 94] with a Vitali type argument so that in the last lemma continuous differentiability of $x \longmapsto v(x ; \xi)$, for real $\xi \in \mathcal{S}(\mathbb{R})$ is sufficient.

\section{Appendix 2: On the Comparison Function}

In this apendix we study properties of the solution $\psi_{a, b}$, the comparison function, of the integral equation $(a, b \in \mathbb{R})$

$$
f(t)=a+b(K f)(t), \quad t \in[0,1],
$$

where $\mathrm{K}$ is the integral operator given by

$$
K f(t)=\int_{0}^{t}(t-s)^{-\frac{1}{2}} f(s) d s .
$$

We will consider $K$ as an operator on $C([0,1])$ (equipped with the sup-norm or an equivalent norm, see below). 
Lemma A.2.1. Let $a, b \in \mathbb{R}$ and set

$$
\psi_{a, b}(t):=a \sum_{n=0}^{\infty} \Gamma\left(\frac{n}{2}+1\right)^{-1}\left(b^{2} \pi t\right)^{\frac{n}{2}}, \quad t \in[0,1]
$$

Then $\psi_{a, b}$ is the unique solution of $(\mathrm{A} .2 .1)$ in $C([0,1])$.

Proof. First of all, note that the right hand side of (A.2.3) converges uniformly in $t \in[0,1]$, and hence it defines a continuous function: $\psi_{a, b} \in C([0,1])$. For $b=0$ it is obvious that $\psi_{a, b}=a$ solves (A.1.1). Let $b \neq 0$, and for $n \in \mathbb{N}_{0}$ consider the integral

$$
\int_{0}^{t}(t-s)^{-\frac{1}{2}} s^{\frac{n}{2}} d s
$$

which is a beta function, and its value is equal to

$$
\sqrt{\pi} \Gamma\left(\frac{n}{2}+1\right) \Gamma\left(\frac{n+1}{2}+1\right)^{-1} t^{\frac{n+1}{2}}
$$

Thus the series

$$
a \sum_{n=0}^{\infty} \Gamma\left(\frac{n}{2}+1\right)^{-1}\left(b^{2} \pi\right)^{\frac{n}{2}} \int_{0}^{t}(t-s)^{-\frac{1}{2}} s^{\frac{n}{2}} d s
$$

converges absolutely and uniformly in $t \in[0,1]$, and Fubini's theorem implies that it is equal to $\left(K \psi_{a, b}\right)(t)$. Hence we have

$$
\begin{aligned}
K \psi_{a, b}(t) & =\frac{a}{b} \sum_{n=0}^{\infty} \Gamma\left(\frac{n+1}{2}+1\right)^{-1}\left(b^{2} \pi t\right)^{\frac{n+1}{2}} \\
& =\frac{1}{b}\left(\psi_{a, b}(t)-a\right)
\end{aligned}
$$

by insertion of the value of the above integral. Thus $\psi_{a, b}$ is a solution of (A.2.1).

If $\varphi, \psi$ are solutions of $(\mathrm{A} .2 .1)$ in $C([0,1])$, then their difference $g \in C([0,1])$ solves

$$
g(t)=b(K g)(t), \quad t \in[0,1],
$$

and thus we have for all $n \in \mathbb{N}, t \in[0,1]$,

$$
g(t)=b^{n}\left(K^{n} g\right)(t)
$$

In particular, we get the estimates

$$
|g(t)| \leq|g|_{\infty}|b|^{n}\left(K^{n} 1\right)(t)
$$

for all $n \in \mathbb{N}, t \in[0,1]$. $\left(K^{n} 1\right)(t)$ can easily be computed by using the Laplace transform, and the result is

$$
\left(K^{n} 1\right)(t)=\Gamma\left(\frac{n}{2}+1\right)^{-1}(\pi t)^{\frac{n}{2}}
$$


Therefore we have for all $n \in \mathbb{N}$,

$$
|g|_{\infty} \leq|g|_{\infty}|b|^{n} \pi^{\frac{n}{2}} \Gamma\left(\frac{n}{2}+1\right)^{-1} .
$$

Consequently $g=0$, i.e., $\psi_{a, b}$ is the unique solution of (A.2.1).

Lemma A.2.2. Let $a, b \in \mathbb{R}$. For all $t \in[0,1]$ the solution $\psi_{a, b}$ of (A.2.1) admits the bounds

$$
|a| e^{t \pi b^{2}} \leq\left|\psi_{a, b}(t)\right| \leq|a|(1+|b| \sqrt{\pi t}) e^{t \pi b^{2}}
$$

Proof: Without loss of generality let $a, b \geq 0$ so that $\psi_{a, b} \geq 0$. Then

$$
\begin{aligned}
\psi_{a, b}(t) & =a \sum_{n=0}^{\infty} \Gamma\left(\frac{n}{2}+1\right)^{-1}\left(t \pi b^{2}\right)^{\frac{n}{2}} \\
& \geq a \sum_{n=0}^{\infty} \Gamma(n+1)^{-1}\left(t \pi b^{2}\right)^{n} \\
& =a e^{t \pi b^{2}}
\end{aligned}
$$

For the upper bound estimate as follows:

$$
\begin{aligned}
\psi_{a, b}(t) & =a\left(e^{t \pi b^{2}}+\sum_{n=0}^{\infty} \Gamma\left(n+\frac{3}{2}\right)^{-1}\left(t \pi b^{2}\right)^{n+\frac{1}{2}}\right) \\
& \leq a\left(1+\left(t \pi b^{2}\right)^{\frac{1}{2}}\right) e^{\ell \pi b^{2}}
\end{aligned}
$$

On. $C([0,1])$ we define a family of norms $\left\{|\cdot|_{b, \infty}, b \geq 0\right\}$ by

$$
|f|_{b, \infty}:=\sup _{t \in[0,1]}\left|f(t) \psi_{1, b}^{-1}(t)\right| \text {. }
$$

By Lemma A.2.2 it is clear that for every $b \geq 0,|\cdot|_{b, \infty}$ is equivalent to the sup-norm $|\cdot|_{\infty}$.

Next we derive a Gronwall type estimate.

Lemma A.2.3. Assume that $f \in C([0,1])$ satisfies for some $b>0$ the inequality

$$
f(t) \leq b(K f)(t), \quad t \in[0,1] .
$$

Then $f(t) \leq 0$ for all $t \in[0,1]$.

Proof. Since $K$ has a positive kernel, (A.2.5) implies $f(t) \leq b\left(K f_{+}\right)(t)$ for all $t \in[0,1]$, where $f_{+}=\max (f, 0) \in C([0,1])$. Therefore $(\mathrm{A} .2 .5)$ yields for all $c>0$,

$$
\begin{aligned}
f_{+}(t) & \leq b\left(K f_{+}\right)(t) \\
& =b\left(K \psi_{1, c} \psi_{1, c}^{-1} f_{+}\right)(t) \\
& \leq b\left|f_{+}\right|_{c, \infty}\left(K \psi_{1, c}\right)(t) \\
& =b\left|f_{+}\right|_{c, \infty} \frac{1}{c}\left(\psi_{1, c}(t)-1\right) \\
& \leq \frac{b}{c}\left|f_{+}\right|_{c, \infty} \psi_{1, c}(t) .
\end{aligned}
$$


Hence we get for all $c>0$,

$$
\left|f_{+}\right|_{c, \infty} \leq \frac{b}{c}\left|f_{+}\right|_{c, \infty}
$$

We only need to choose $c=2 b$ to conclude $\left|f_{+}\right|_{c, \infty}=0$, which entails $f_{+}=0$.

Lemma A.2.4. Let $f \in C([0,1])$ be non-negative and satisfy

$$
f(t) \leq a+b(K f)(t), \quad t \in[0,1],
$$

for $a \geq 0, b \geq 0$. Then

$$
f(t) \leq \psi_{a, b}(t) \leq a(1+b \sqrt{\pi t}) e^{t \pi b^{2}}, \quad t \in[0,1] .
$$

Proof. We assume that $a, b>0$, otherwise the statement is trivial. Then we have by hypothesis

$$
f(t)-\psi_{a, b}(t) \leq b K\left(f-\psi_{a, b}\right)(t) .
$$

By Lemma A.2.3, $f(t) \leq \psi_{a, b}(t)$ for all $t \in[0,1]$.

Remark. Of course, estimate (A.2.7) can also be derived by iteration of (A.2.6), and an application of the Laplace transform to compute the multiple convolution integrals.

ACKNOWLEDGEMENT. It is a pleasure to thank G. Cochran and J.-S. Lee for fruitful discussions.

References.

[BT 94]

[CL 93]

[Ch 89]

[DP 94]

[Fr 83]

[HK 93]

[KL 94]

[PS 91]
Benth, F. and Timpel, M.: Topological aspects of the characterization of Hida distributions - A remark; Preprint (1994)

Cochran, G., Lee, J.-S. and Potthoff, J.: Stochastic Volterra equations with singular kernels; Preprint (1993)

Chow, P.L.: Generalized solution of some parabolic equations with a random drift; J. Appl. Math. Optimization 20 (1989) 81-96

Deck, Th. and Potthoff, J.: Parabolic stochastic partial differential equations; in preparation

Friedman, A.: Partial Differential Equations of Parabolic Type. Malabar: Krieger (1983)

Hida, T., Kuo, H.-H., Potthoff, J. and Streit, L.: White Noise - An Infinite Dimensional Calculus. Dordrecht: Kluwer (1993)

Kondratiev, Yu.G., Leukert, P., Potthoff; J., Streit, L. and Westerkamp, W.: Generalized functionals in Gaussian spaces - The characterization theorem revisited; Preprint (1994)

Potthoff, J. and Streit, L.: A characterization of Hida distributions; J. Funct. Anal. 101 (1991) 212-229 